\title{
Optimal Investment in the Development of Oil and Gas Field ${ }^{\star}$
}

\author{
Adil Erzin ${ }^{1,2[0000-0002-2183-523 X]}$, Roman Plotnikov ${ }^{1[0000-0003-2038-5609]}$, \\ Alexei Korobkin ${ }^{3}$, Gregory Melidi² ${ }^{2}$, and Stepan Nazarenko ${ }^{2}$ \\ 1 Sobolev Institute of Mathematics, SB RAS, Novosibirsk 630090, Russia \\ 2 Novosibirsk State University, Novosibirsk 630090, Russia \\ ${ }^{3}$ Gazpromneft, St. Petersburg, Russia \\ adilerzin@math.nsc.ru
}

\begin{abstract}
Let an oil and gas field consists of clusters in each of which an investor can launch at most one project. During the implementation of a particular project, all characteristics are known, including annual production volumes, necessary investment volumes, and profit. The total amount of investments that the investor spends on developing the field during the entire planning period we know. It is required to determine which projects to implement in each cluster so that, within the total amount of investments, the profit for the entire planning period is maximum.

The problem under consideration is NP-hard. However, it is solved by dynamic programming with pseudopolynomial time complexity. Nevertheless, in practice, there are additional constraints that do not allow solving the problem with acceptable accuracy at a reasonable time. Such restrictions, in particular, are annual production volumes. In this paper, we considered only the upper constraints that are dictated by the pipeline capacity. For the investment optimization problem with such additional restrictions, we obtain qualitative results, propose an approximate algorithm, and investigate its properties. Based on the results of a numerical experiment, we conclude that the developed algorithm builds a solution close (in terms of the objective function) to the optimal one.
\end{abstract}

Keywords: Investment portfolio optimization · Production limits.

\section{Introduction}

The founder of the mathematical theory of portfolio optimization is G. Markowitz, who, in 1952, published an article [16] with the basic definitions and approaches for evaluating investment activity. He developed a methodology for the formation of an investment portfolio, aimed at the optimal choice of assets, based on a given ratio of profitability/risk. The ideas formulated by him form the basis of modern portfolio theory [16 17].

\footnotetext{
* The research is carried out within the framework of the state contract of the Sobolev Institute of Mathematics (project 0314-2019-0014).
} 
The author of [21] gave a review of portfolio selection methods and described the prospects of some open areas. At first, the author described the classical Markowitz model. Then comes the "intertemporal portfolio choice" developed by Merton [1819], the fundamental concept of dynamic hedging and martingale methods. Pliska [21, Karatzas [11, as well as Cox and Huang [6] made the main contribution to the development of this direction. The authors of [7] and [20] proposed the formulas for the optimal portfolio for some private productions. These formulas have the form of conditional expectation from random variables.

In most well-known studies, the problem of optimal investment is solved numerically [34], which does not allow us to identify the contribution of portfolio components to the optimal solution. In 2, a new approach is proposed for dynamic portfolio selection, which is not more complicated than the Markowitz model. The idea is to expand the asset space by including simple, manageable portfolios and calculate the optimal static portfolio in this extended space. It is intuitively assumed that a static choice among managed portfolios is equivalent to a dynamic strategy.

If we consider investing in specific production projects, then each of them is either implemented or not. In contrast to the classical Markowitz's problem, a discrete statement, arises, and the mathematical apparatus developed for the continuous case is not applicable. In [15, the authors examined a two-criterion problem of maximizing profit and minimizing risk. The characteristics of each project, mutual influence, and the capital available to the investor are known. For the Boolean formulation of the problem, the authors proved NP-hardness and found special cases when the problem is solved with pseudopolynomial time complexity.

The portfolio optimization problems described above relate to the stock market. For companies operating in the oil and gas sector, optimization problems are relevant. In these problems it is necessary to maximize total profit and minimize risks for a given period, taking into account additional restrictions, for example, on production volume, as well as problems in which it is necessary to maximize production (or profit) for a given amount of funding. In [1, the author presented an approach aimed at improving the efficiency of the management of the oil and gas production association. Two control loops are distinguished: macroeconomic, which is responsible for optimizing policies at the aggregated level (industry and regional), and microeconomic, which is responsible for optimizing the organizational and functional structure of the company. The first circuit implemented using the author developed computable models of general economic equilibrium and integrated matrices of financial flows. The second circuit performed using an approach based on simulation of the business processes of an enterprise.

The author of 9 considers the problem of forming a portfolio of investment projects, which required to obtain maximum income under given assumptions regarding risks. A method is proposed based on a comprehensive multidimensional analysis of an investment project. The authors of [1012 consider a problem of minimizing the deposit costs with restrictions on the volume of the production. They propose an algorithm for building an approximate solution by dynamic 
programming. The accuracy of the algorithm depends on the discretization step of the investment volume. The authors of [10] formulate the problem of minimizing various costs associated with servicing wells, with limitations related to the amount of oil produced, as a linear programming problem, and find the optimal solution using the simplex method.

For the decision-maker, the main concern is how to allocate limited resources to the most profitable projects. Recently, a new management philosophy, Beyond NPV (Net Present Value), has attracted more and more international attention. Improved portfolio optimization model presented in [23]. It is an original method, in addition to NPV, for budgeting investments. In the proposed model, oil company executives can compromise between profitability and risk concerning their acceptable level of risk. They can also use the "operating bonus" to distinguish their ability to improve the performance of major projects. To compare optimized utility with non-optimized utility, the article conducted a simulation study based on 19 foreign upstream assets owned by a large oil company in China. The simulation results showed that the optimization model, including the "operating bonus", is more in line with the rational demand of investors.

The purpose of the paper [5] is to offer a tool that might support the strategic decision-making process for companies operating in the oil industry. Their model uses Markowitzs portfolio selection theory to construct an efficient frontier for currently producing fields and a set of investment projects. These relate to oil and gas exploration projects and projects aimed at enhancing current production. The net present value obtained for each project under a set of usersupplied scenarios. For the base-case scenario, the authors also model oil prices through Monte Carlo simulation. They run the model for a combination of portfolio items, which include both currently producing assets and new exploration projects, using data characteristics of a mature region with a high number of low-production fields. The objective is to find the vector of weights (equity stake in each project), which minimizes portfolio risk, given a set of expected portfolio returns.

Due to the suddenness, uncertainty, and colossal loss of political risks in overseas projects, the paper [14 considers the time dimension and the success rate of project exploitation for the goal of optimizing the allocation of multiple objectives, such as output, investment, efficiency, and risk. A linear portfolio risk decision model proposed for multiple indicators, such as the uncertainty of project survey results, the inconsistency of project investment time, and the number of projects in unstable political regions. Numerical examples and the results test the model and show that the model can effectively maximize the portfolio income within the risk tolerance range under the premise of ensuring the rational allocation of resources.

This paper discusses the problem of optimal investment of oil and gas field development consisting of subfields - clusters. For each cluster, there are several possibilities for its development, which we call the projects. Each project characterized by cost, lead time, resource intensity, annual production volumes, and profit from its implementation. Also, there are restrictions on the annual 
production volumes of the entire field. This requirement leads to the need for a later launch of some projects so that the annual production volume does not exceed the allowable volumes. Assuming that a project launched later is another project, we proposed a statement of the problem in the form of a Boolean linear programming (BLP) problem. We estimated the maximum dimension at which CPLEX solves the BLP in a reasonable time. For a large-dimensional problem, we developed a method that constructs an approximate solution in two stages. At the first stage, the problem is solved without limitation on the volume of annual production. This problem remains NP-hard, but it is solvable by a pseudopolynomial dynamic programming algorithm. As a result, one project is selected for each cluster. The project is characterized, in particular, by the year of launch and production volumes in each subsequent year. If we start the project later, the annual production volumes shift. In the second stage, the problem of determining the start moments of the projects selected at the first stage is solved, taking into account the restrictions on annual production volumes, and the profit is maximal. We developed a local search algorithm for partial enumeration of permutations of the order in which projects are launched. At the same time, for each permutation, the algorithm of tight packing of production profiles developed by us (we call it a greedy algorithm), which builds a feasible solution, is applied. A numerical experiment compared our method and CPLEX.

The rest of the paper has the following organization. In Section 2, we state the problem as a BLP. In Section 3, the problem without restrictions on the volume of production reduced to a nonlinear distribution problem, which is solved by dynamic programming. As a result, a "best" project found for each cluster. Section 4 describes the method for constructing an approximate solution by searching for the start times for the "best" projects. The next section presents the results of a numerical experiment. We identify the maximum dimension of the problem, which is solved by the CPLEX package in a reasonable time, and compare the accuracy of the developed approximate algorithms. Section 6 contains the main conclusions and describes the directions for further research.

\section{Formulation of the problem}

For the mathematical formulation of the problem, we introduce the following notation for the parameters:

- $[1, T]$ is the planning period;

$-C$ is the total amount of investment;

$-K$ is the set of clusters $(|K|=n)$;

$-P_{k}$ is the set of projects for the development of the cluster $k \in K\left(\max \left|P_{k}\right|=\right.$ $p$ ) taking into account the shift at the beginning of each project;

$-d_{k}^{i}(t)$ is the volume of production in the cluster $k \in K$ per year $t=1, \ldots, T$, if the project $i \in P_{k}$ is implemented there;

$-q_{k}^{i}$ is the profit for the entire planning period from the implementation of project $i$ in cluster $k$; 
$-c_{k}^{i}$ is the cost of implementing project $i$ in cluster $k$;

- $D(t)$ is the maximum allowable production per year $t$;

and for the variables:

$$
x_{k}^{i}=\left\{\begin{array}{l}
1, \text { if project } i \text { is selected for cluster } k \\
0, \text { else. }
\end{array}\right.
$$

Then the problem under consideration can be written as follows.

$$
\begin{gathered}
\sum_{k \in K} \sum_{i \in P_{k}} q_{k}^{i} x_{k}^{i} \rightarrow \max _{x_{k}^{i} \in\{0,1\}} ; \\
\sum_{k \in K} \sum_{i \in P_{k}} c_{k}^{i} x_{k}^{i} \leq C ; \\
\sum_{i \in P_{k}} x_{k}^{i} \leq 1, k \in K ; \\
\sum_{k \in K} \sum_{i \in P_{k}} d_{k}^{i}(t) x_{k}^{i} \leq D(t), t \in[1, T] .
\end{gathered}
$$

Remark 1. Each project has various parameters, among which the annual production volumes. If we start the project later, then the graphic of annual production will shift entirely. Suppose $d_{k}^{i}(t)$ is the volume production per year $t$ if the project $i$ is implemented in the cluster $k$. If this project is launched $\tau$ years later, the annual production during year $t$ will be $d_{k}^{i}(t-\tau)$. So, each project in the set $P_{k}$ is characterized, in particular, by its beginning.

However, not all characteristics retain their values at a later launch of the project. Profit from project implementation depends on the year of its launch, as money depreciates over the years. One way to account for depreciation is to use a discount factor. The value of money decreases with each year by multiplying by a discount factor that is less than 1 . In this regard, at the stage of preliminary calculations, we recount values associated with investment and profit.

As a result, the set $P_{k}$ consists of the initial projects, and the shifted projects for different years as well. So, having solved the problem (11)-(4), we will choose for each cluster not only the best project but also the time of its start.

Problem (11)-(41) is an NP-hard BLP. For the dimension which we define in Section 5, a software package, for example, CPLEX, can be used to solve it. In order to solve the problem of a large dimension, it is advisable to develop an approximate algorithm. To do this, in the next section we consider the problem (1) $-(3)$.

\section{The problem without restrictions on production volumes}

If there are no restrictions on production volumes, then instead of the variables $x_{k}^{i}$, we can use the variables $c_{k}$, which are equal to the amount of the investment 
allocated for the development of the cluster $k$. To do this, for each cluster $k$, we introduce a new profit function $q_{k}\left(c_{k}\right)$, which does not depends on the selected project but depends on the amount of investment. For each $k$, the function $q_{k}\left(c_{k}\right)$ is obviously non-decreasing piecewise constant. Moreover, if we know the value $c_{k}$, then one project is uniquely will be used to develop the cluster $k$, and all its characteristics will be known. Indeed, the more money is required to implement the project, the more efficient it is (more profitable). If this is not a case, then a less effective but more expensive project can be excluded. Obviously, the values of all functions $q_{k}\left(c_{k}\right), c_{k} \in[0, C], k \in K$, are not difficult to calculate in advance. The complexity of this procedure does not exceed $O(K p C)$.

Given the previous, we state the problem of maximizing profit without restrictions on production volumes, assuming that all projects start without delay, in the following form.

$$
\begin{gathered}
\sum_{k \in K} q_{k}\left(c_{k}\right) \rightarrow \max _{c_{k} \in[0, C]} ; \\
\sum_{k \in K} c_{k} \leq C .
\end{gathered}
$$

Although problem (5)-(6) become easier than the problem (10)-(4), it remains NP-hard. However, it is a distribution problem, for the solution of which we apply the dynamic programming method, the complexity of which is $O\left(n(C / \delta)^{2}\right)$, where $\delta$ is the step of changing the variable $c_{k}$. Solving the problem (5)-(6), we choose the "best" project for each cluster. If it turns out that at the same time, all the restrictions (44) fulfilled, then this solution is optimal for the original problem (11)-(4). If at least one inequality (4) violated, then we will construct a feasible solution in the manner described in the next section.

\section{Consideration of restrictions on production volumes}

We will not change the projects selected for each cluster as a result of solving the problem (5)-(6). We will try to determine the moments of launching these projects so that inequalities (4) fulfill, and profit takes maximal value. The project selected for the cluster $k$ is characterized by the production volumes $d_{k}(t)$ in each year $t \in[1, T]$. It is necessary to shift the beginning of some projects to a later time so that in each year $t \in[1, T]$ the total production is at most $D(t)$ :

$$
\sum_{k \in K} d_{k}(t) \leq D(t) .
$$

Assume that the cluster $k$ development project, whose beginning is shifted by $i \in\left[0, t_{k}\right]$ years, is another project. Then for each cluster, there is a set of projects, which we denote as before by $P_{k}\left(\left|P_{k}\right|=t_{k}+1\right)$. As a result, to determine the shift in the start of the project launch for each cluster, it is enough to solve the problem (11) -(4) without restriction (2), in which the Boolean variable $x_{k}^{i}=1$ if and only if the start of the cluster project $k$ is shifted by $i$ years. Then the solution to the small-dimension problem can be found using a CPLEX. However, for a large-sized problem, it is necessary to use an approximate algorithm. 


\subsection{Greedy algorithm}

Suppose we order the projects according to the years of their launch. A shift in the start of projects changes this order. The order in which projects start uniquely determined by the permutation $\pi$ of the cluster numbers $\{1,2, \ldots, n\}$. For a given permutation, we describe informally a greedy algorithm that constructs a feasible solution to the problem.

Denote by $P(\pi)$ the list of ordered projects. The first project starts without delay (with zero shift). We exclude it from the set $P(\pi)$. For the first project of the updated set $P(\pi)$, we determine its earliest start time, which is no less than the start time of a previous project, to comply with the production order and restrictions (4) in each year and exclude this project from the set $P(\pi)$. We continue the process until the start year of the last project, $\pi(n)$ is found.

The greedy algorithm will construct a feasible solution for the given permutation $\pi$, if it exists, with the time complexity of $O(n T)$. In the oil and gas industry, profiles (graphs) of annual production volumes have a log-normal distribution 22, which is characterized by a rapid increase, and then a slight decrease. This observation and the following lemma, to some extent, justify why we use the greedy algorithm.

Proposition 1. If the order of launching the projects is known, the annual production schedules for all projects are not-increasing, and $D(t)=D=$ const, $t \in[1, T]$, then the greedy algorithm determines the optimal start years for all projects.

Proof. In the problem under consideration, time is discrete (measured in years). Therefore, the value of production in each cluster is a certain real number that does not change for one year. A greedy algorithm for a given order of projects determines the earliest start time for each project, which is not less than the start time of the previous project. Suppose that in all optimal solutions, there is at least one project that begins later than the year determined by the greedy algorithm. Consider some optimal solution and let $k$ be the first project that we can start earlier (Fig. 1 $1 a$ ). Since the project $k$ can start earlier, then move it as much as possible to the left to maintain validity (Fig. 1b). Notice that it is enough to check the value of production $d_{k}^{1}$ only in the first year of the project $k$ because it is not less than production in subsequent years $\left(d_{k}^{t} \leq d_{k}^{1}, t>1\right)$. The solution obtained after shifting the project $k$ to the left is no worse (and taking into account the discount coefficient, even better), but the project $k$ starts earlier, which contradicts the assumption. The proof is over.

So, with a particular order of projects, a greedy algorithm builds a solution close to optimal with $O(n T)$ time complexity. A complete enumeration of permutations requires $O(n$ !) operations. However, it is reasonable to develop a local search algorithm in which, at each step, the best permutation is searched in the vicinity of the current permutation. In order to obtain a solution for a given permutation, a greedy algorithm is used. The higher the profit in a particular order of projects, the better the permutation. In the next subsection, we develop a local search algorithm. 


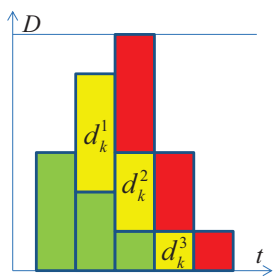

a)

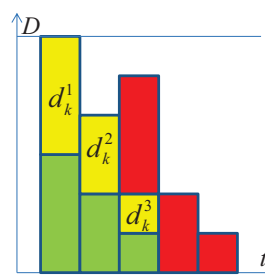

b)

Fig. 1. Illustration to the Lemma 1 proof (Bars of the same color belong to the same project, and the height of the bar is the volume of production in the corresponding year). a) Project $k$ (yellow) may start earlier; $b$ ) After shifting the project $k$ to the left.

\subsection{Local search}

Using the greedy algorithm described in the previous subsection, one can construct a solution for each permutation of the cluster numbers. Therefore, it is essential to find a permutation where the solution constructed in such a way is near-optimal. For this reason, we suggest a local search procedure for permutations starting from some promising one.

In order to obtain the first permutation for the local search procedure we perform the following greedy algorithm. At the first step we choose such cluster that yields maximum value of income if its development is started at the first year. The number of this cluster becomes the first value of the permutation. Then, at each step of the algorithm, we choose among the unprocessed clusters such cluster, that if its development is started at the earliest year (taking into account the per-year production bound and already chosen clusters), then the total income increment will be maximum. After such cluster is found, we assign the corresponding shift for it and set its number to the next permutation value. The permutation obtained by the described greedy procedure becomes the first permutation of the local search algorithm.

For each permutation in the local search procedure, we construct a solution using the greedy algorithm, that is described in previous subsection, with time complexity $O(n T)$. As a movement operation of the local search procedure, we perform the best possible exchange of two different elements of a permutation. Then the cardinality of the neighborhood of the current permutation is $O\left(n^{2}\right)$, and the time complexity of the searching the best solution is this neighborhood is $O\left(n^{3} T\right)$.

\section{Simulation}

The proposed algorithms have been implemented in the $\mathrm{C}++$ programming language and launched on the randomly generated test instances. We also used the IBM ILOG CPLEX package (version 12.10) in order to obtain optimal or nearoptimal solutions together with guaranteed upper bounds for the comparison. 
The numerical experiment performed on an Intel Core i5-4460 (3.2GHz) $8 \mathrm{~Gb}$ machine.

For the generation of the test data, we supposed that the distribution function of production volumes by the planned period is log-normal 22. The parameters of the distribution density $\mu$ and $\sigma$ were chosen randomly with uniform distribution on the intervals $[1,2]$ and $[1,1.4]$ correspondingly. We defined the maximum value of the production volume for each project at random with uniform distribution on the interval $[30,200]$ (in thousands of tons) and then multiplied each per-year volume by the corresponding scaling factor.

We assumed that the profit is proportional to the production volume. For each project, in order to generate the profit per each year, we, at first, took the random coefficient (the cost of one ton in millions of rubles) uniformly distributed on the interval $[4,6]$. This value may vary depending on the differences in the condition of the production, overhead costs, remoteness of the cluster. After that, we multiplied the per-year production volumes by this coefficient. We also added the noise to the generated values multiplying them by the random values uniformly distributed on the interval $[0.95,1.05]$.

Like the investments, we generated random values uniformly distributed on the interval $[250,1500]$ (in millions of rubles). We assumed that the obtained amount of money spent in the first year of the project exploitation. In about 10 percent of the cases, other investments made in the second year of the project exploitation. The second investment is taken as a random part of the first investment from 10 to 50 percent. As the upper bounds of investments and per-year production volumes, we took the one-third part of the sum by clusters of the maximum values per project and per year. That is,

$$
C=1 / 3 \sum_{k \in K} \max _{i \in P_{k}} c_{k}^{i}
$$

and

$$
D=1 / 3 \sum_{k \in K} \max _{i \in P_{k}, t \in[1, T]} d_{k}^{i}(t), D(t)=D \forall t \in[1, T] .
$$

For solving the problem (5)-(6) we set $\delta$ equal ten thousands of rubles, because, according to our preliminary experiments, further decrease of $\delta$ does not improve the solution significantly.

We generated instances for four different variants of the number of clusters: $n=10,25,50$, and 100. For each value of $n$, we generated four instances with different maximum and a minimum number of projects per cluster: 1) from 1 to 10; 2) from 10 to $25 ; 3)$ from 25 to 50 , and 4) from 50 to 100 . We launched our algorithm and CPLEX on each instance. The results presented in Table 11 In this table, CPLEX stands for the results obtained by CPLEX launched on the problem (11)-(4). $C P L E X_{f p}$ stands for the results obtained by CPLEX launched on the restricted problem with the fixed project per each cluster found by the dynamic programming method described in Section 3. Notations $o b j, u b$, and $g a p$ stand for, correspondingly, the objective function of the incumbent, the upper bound of the value of objective function, and the relative difference between $o b j$ 
and $u b$. decline stands for the decline (in percents) of the objective function value of the incumbent of the problem with the fixed set of projects concerning the objective function of the incumbent of the entire problem. The last four columns represent the results obtained by our algorithm, which is named $A$ in the table. $r_{1}$ denotes the ratio $\operatorname{obj}(A) / u b(C P L E X)$, and $r_{2}$ denotes the ratio $o b j(A) / u b\left(C P L E X_{f p}\right)$. The last column stands for the total running time of our algorithm. The running time of CPLEX was limited by 60 seconds for all the cases except the last one of the largest size, - in the last case CPLEX was given for 1 hour. It also should be noted that CPLEX was parallelized on four threads.

As it follows from the table, in the cases of small and moderate size CPLEX solves the problem rather precisely within 60 seconds. In these cases it always constructs a solution on which the value of the objective function differs from the optimal one by at most 1 percent. Algorithm $A$ constructs a less accurate solution. As it is seen at the column $r_{1}$, in the worst case, the objective value of the obtained solution differs from the optimal by 13 percent, in the best case by 3 percent, and on average, this difference does not exceed 9 percent. As one can see at the column decline, the choice of the projects obtained by solution of the problem without restriction on the production volumes deteriorates the solution of the entire problem by up to 12.5 percent. On average, this decline is about 6 percent. The quality of our local search procedure applied to the solution obtained by the greedy heuristic is estimated in the column $r_{2}$. On average, the ratio does not exceed 3 percent. In a case of large size, when the number of clusters is 250 and the number of projects in each cluster varies from 250 to 500 , CPLEX failed to construct any feasible solution within 1 hour, but the algorithm $A$ constructed an approximate solution within about 6 minutes. When we set the projects found by algorithm $A$ to CPLEX for this instance, it successfully found the solution with rather small value of gap (less than 0.1 percent) within 1 hour. In this case, the local search procedure found a solution that differs from the optimal one by not more than 4 percent.

\section{Conclusion}

In this paper, we studied the NP-hard problem of maximizing profit by choosing long-term cluster development projects within the oil and gas field, with restrictions on the total investment and maximum annual production. We proposed a statement of the problem in the form of Boolean linear programming and set ourselves three goals. First, to investigate the effectiveness of application software packages, such as CPLEX, for solving the BLP problem. Secondly, develop a fast approximate algorithm. Thirdly, compare the effectiveness of the CPLEX package and the approximate algorithm.

The approximate algorithm consists of two stages, which are partially dictated by the specifics of the problem. At the first stage, profit is maximized by selecting one project for each cluster without taking into account the restrictions on the volume of annual production. The distribution problem arising, in this 


\begin{tabular}{|c|c|c|ccc|cccc|cccc|}
\hline \multirow{2}{*}{$n$} & $p_{\min }$ & $p_{\max }$ & \multicolumn{3}{|c|}{$C P L E X$} & \multicolumn{4}{c|}{$C P L E X_{f p}$} & \multicolumn{4}{c|}{$A$} \\
& & & $o b j$ & $u b$ & $g a p$ & $o b j$ & $u b$ & $g a p$ & decline (\%) & obj & $r_{1}$ & $r_{2}$ & time (sec.) \\
\hline \multirow{5}{*}{10} & 1 & 10 & 12851.93 & 12851.93 & 0 & 12072 & 12072 & 0 & 6.07 & 12072 & 0.94 & 1 & 0.006 \\
& 10 & 25 & 16460.95 & 16460.95 & 0 & 15502.23 & 15502.23 & 0 & 5.82 & 14710.6 & 0.89 & 0.95 & 0.005 \\
& 25 & 50 & 16988.53 & 16988.53 & 0 & 14862.28 & 14862.28 & 0 & 12.51 & 14764.3 & 0.87 & 0.99 & 0.004 \\
& 50 & 100 & 17140.36 & 17140.36 & 0 & 15607.92 & 15607.92 & 0 & 8.94 & 15374.1 & 0.9 & 0.99 & 0.003 \\
\hline \multirow{5}{*}{25} & 1 & 10 & 30465.26 & 30465.26 & 0 & 29849.9 & 29849.9 & 0 & 2.02 & 29571.9 & 0.97 & 0.99 & 0.082 \\
& 10 & 25 & 42501.57 & 42501.57 & 0 & 39728.94 & 39728.94 & 0 & 6.52 & 38930.6 & 0.92 & 0.98 & 0.077 \\
& 25 & 50 & 46508.15 & 46849.94 & 0.007 & 43646.48 & 43646.48 & 0 & 6.15 & 42407.4 & 0.9 & 0.97 & 0.056 \\
& 50 & 100 & 47432.09 & 47906.72 & 0.01 & 44307.47 & 44307.47 & 0 & 6.59 & 43324 & 0.9 & 0.98 & 0.023 \\
\hline \multirow{5}{*}{50} & 1 & 10 & 70568.99 & 70568.99 & 0 & 69609.18 & 69609.18 & 0 & 1.36 & 66328.2 & 0.94 & 0.95 & 0.752 \\
& 10 & 25 & 86529.19 & 86659.92 & 0.002 & 80616.31 & 80778.51 & 0.002 & 6.83 & 76845.5 & 0.89 & 0.95 & 0.627 \\
& 25 & 50 & 93928.28 & 94290.32 & 0.003 & 88415.05 & 88415.05 & 0 & 5.87 & 86861.8 & 0.92 & 0.98 & 0.612 \\
& 50 & 100 & 95201.48 & 95621.93 & 0.004 & 88532.86 & 88661.56 & 0.001 & 7 & 86380.7 & 0.9 & 0.97 & 0.39 \\
\hline \multirow{5}{*}{100} & 1 & 10 & 139928.34 & 140023.11 & 0.0007 & 136420.24 & 136586.12 & 0.001 & 2.51 & 128679 & 0.92 & 0.94 & 5.22 \\
& 10 & 25 & 173898.61 & 174065.77 & 0.001 & 163833.54 & 163932.06 & 0.0006 & 5.79 & 154452 & 0.89 & 0.94 & 5.35 \\
& 25 & 50 & 189722.3 & 190000.79 & 0.001 & 177064.12 & 177138.3 & 0.0004 & 6.67 & 171431 & 0.9 & 0.97 & 5.84 \\
& 50 & 100 & 195223.21 & 195686.39 & 0.0023 & 180375.66 & 180476.58 & 0.0005 & 7.61 & 176830 & 0.9 & 0.98 & 9.44 \\
\hline 250 & 250 & 500 & - & - & - & 515525.8 & 516003.7 & 0.0009 & - & 495366 & - & 0.96 & 366.4 \\
\hline
\end{tabular}

Table 1. Comparison of the proposed algorithm $A$ with CPLEX

case, is solved by the dynamic programming algorithm with acceptable running time. Projects selected at the first stage can be launched later (with a delay). Therefore, at the second stage, the moments of the start of the selected projects are determined in such a way that the annual production volumes do not exceed the set values, and the profit is maximum. The problem of the second stage also formulated in the form of the BLP. It makes sense without the first stage because, in practice, development projects for each cluster often known, and it is only necessary to determine the moments of their launch.

Production profiles have a characteristic shape, which is determined by the log-normal distribution law and has the form of a graph that first overgrows, reaches its maximum value, and then slowly decreases [22]. With a certain degree of assumption, we assumed that the profiles are non-increasing. We proved that in the case of non-increasing production profiles and for a given order of project start (which is determined by the permutation of cluster numbers), the greedy algorithm constructs the optimal solution. The algorithm of permutations sorting is justified, and the greedy algorithm used for each permutation. Iterating over all permutations is time-consuming, and for large dimensions, it is just not applicable, so we used a relatively simple local search algorithm.

The results of the numerical experiment on randomly generated examples surprised us (see Table 1). For $25 \leq n \leq 100$, the CPLEX package was not able to build an optimal solution, but it turned out that CPLEX within one minute builds a feasible solution quite close to the optimal one. The approximate algorithm that we developed also builds a solution close to optimal, but CPLEX turned out to be more efficient for such dimension. Thus, we conclude that for the considered problem when $n \leq 100$, it is advisable to use a package of application programs CPLEX instead of our algorithm. In a case of large size, for example when $n \geq 250$, CPLEX failed to construct any feasible solution 
within 1 hour, but the algorithm $A$ constructed an approximate solution within 6 minutes. When we set the projects found by solving the problem (5)-(6) with $n=250$ to CPLEX, it successfully found the solution with gap less than $0.1 \%$ within 1 hour.

Perhaps the situation will change if we consider some additional restrictions. In practice, it is necessary to produce annually at least a given volume and no more than a predetermined quantity. Moreover, there are restrictions on the size of annual investments. Furthermore, annual production volumes are random variables, so the need to take into account the probabilistic nature of the source data can ruin the problem so that the use of CPLEX will become inappropriate.

In future research, we plan to take into account the additional restrictions and specifics, as well as to develop a more efficient approximate algorithm based on a genetic algorithm in which an effective local search, for example, VNS [13, will be used at the mutation stage.

\section{References}

1. Akopov, A.S.: Metodi povisheniya effektivnosti upravleniya neftegazodobivaiushimi ob'edinenuyami. Ekonomicheskaya nauka sovremennoy Rossii.4, 88-99 (2004) (in Russian)

2. Brandt, , M.W., Santa-Clara, P.: Dynamic Portfolio Selection by Augmenting the Asset Space. NBER Working Paper. 10372, JEL No. G0, G1 (2004)

3. Brandt, M.W., Goyal, A., Santa-Clara, P., Stroud, J.R.: A simulation approach to dynamic portfolio choice with an application to learning about return predictability. Rev. Financ. Stud. 18, 831-873 (2005)

4. Brennan, M., Schwartz, E., Lagnado, R.: Strategic asset allocation. J. Econ. Dyn. Control. 21, 1377-1403 (1997)

5. Bulai, V.C., Horobet, A.: A portfolio optimization model for a large number of hydrocarbon exploration projects. In: Proc. 12 Int. Conf. on Business Excellence. 12(1), 171- 181 (2018). https://doi.org/10.2478/picbe-2018-0017

6. Cox, J.C., Huang, C.-F.: Optimal consumption and portfolio policies when asset prices follow a diffusion process. J. Econ. Theory 49, 33-83 (1989)

7. Detemple, J.B., Garcia, R., Rindisbacher, M.: A Monte-Carlo method for optimal portfolios. J. Finance. 58, 401-446 (2003)

8. Detemple, J.: Portfolio Selection: A Review. J. Optim. Theory Appl. 161, 1-21 (2014)

9. Dominikov, A., Khomenko, P., Chebotareva, G., and Khodorovsky, M.: Risk and profitability optimization of investments in the oil and gas industry. Int. J. of Energy Production and Management. 2(3), 263-276 (2017)

10. Goncharenko, S.N., Safronova, Z.A.: Modeli i metody optimizacii plana dobychi i pervichnoy pererabotki nifti. Gorniy informacionno-analiticheskiy biulleten'. 10, 221-229 (2008) (in Russian)

11. Karatzas, I., Lehoczky, J.P., Shreve, S.E.: Optimal portfolio and consumption decisions for a "mall investor" on a finite horizon. SIAM J. Control Optim. 25, 1557-1586 (1987)

12. Konovalov, E.N., Oficerov, V.P., Smirnov, S.V.: Povishenie effectivnosti investiciy $\mathrm{v}$ neftedobyche na osnove modelirovaniya. Problemi upravleniya i modelirovaniya $\mathrm{v}$ slognih sistemsh: Trudi V negdunarodnoy konferencii. Samara. 381-385 (2006) (in Russian) 
13. Hansen, P., Mladenovic, N.: Variable neighborhood search: Principles and applications, European Journal of Operational Research. 130, 449-467 (2001)

14. Huang, S.: An Improved Portfolio Optimization Model for Oil and Gas Investment Selection Considering Investment Period. Open Journal of Social Sciences. 7, 121129 (2019)

15. Malah, S.A., Servah, V.V.: O slognosti zadachi vybora investicionnih proektov. Vestnik Omskogo universiteta. 3, 10-15 (2016) (in Russian)

16. Markowitz, H.M.: Portfolio Selection. J. of Finance. 7(1), 71-91 (1952)

17. Markowitz, H.M.: Portfolio Selection: Efficient Diversification of Investment. - Wiley, New York, 1959

18. Merton, R.C.: Lifetime portfolio selection under uncertainty: the continuous time case. Rev. Econ. Stat. 51, 247-257 (1969)

19. Merton, R.C.: Optimum consumption and portfolio rules in a continuous-time model. J. Econ. Theory 3, 273-413 (1971)

20. Ocone, D., Karatzas, I.: A generalized Clark representation formula, with application to optimal portfolios. Stoch. Stoch. Rep. 34, 187-220 (1991)

21. Pliska, S.: A stochastic calculus model of continuous trading: optimal portfolios. Math. Oper. Res. 11, 371-382 (1986)

22. Power, M. Lognormality in the observed size distribution of oil and gas pools as a consequence of sampling bias. Math Geol 24, 929-945 (1992). https://doi.org/10.1007/BF00894659

23. Qing, X., Zhen, W., Sijing, L., Dong, Z.: An improved portfolio optimization model for oil and gas investment selection. Pet. Sci. 11, 181-188 (2014)

24. Skopina, L.V., Shubnikov, N.E.: Metodicheskiy podhod k ocenke ionvesticionnih proektov v neftedobyche v usloviyah neopredelennosti i riskov. Vestnik NGU. Seriya: Social'no-ekonomicheskie nauki. 14(2), 24-37 (2014) (in Russian) 